\section{Data-independent acquisition}

B. Güssregen

Merck KGaA, Darmstadt, Deutschland

Synonym(e) DIA; SWATH Acquisition

Englischer Begriff data-independent acquisition
Definition Eine häufig in der Proteinanalytik angewandte Messmethode der $\triangleright$ Massenspektrometrie, bei der alle Ionen innerhalb eines ausgewählten $\mathrm{m} / \mathrm{z}$-Bereichs in einem zweiten Scan im Tandem-Massenspektrometer fragmentiert und analysiert werden.

Beschreibung MS/MS-Spektren werden entweder durch Fragmentierung aller Ionen, die zu einem gegebenen Zeitpunkt in das Massenspektrometer eintreten (sog. BreitbandDIA) oder durch vordefinierte Bereiche von $\mathrm{m} / \mathrm{z}$ erhalten. 